\title{
HOW LONG DOES IT TAKE TO RELAX? OBSERVATION OF DRIVER BEHAVIOR DURING REAL-WORLD CONDITIONALLY AUTOMATED DRIVING
}

\author{
Kamil Omozik ${ }^{1,2}$, Yucheng Yang ${ }^{2}$, Isabella Kuntermann ${ }^{1}$, Sebastian Hergeth ${ }^{1}$, Klaus Bengler ${ }^{2}$ \\ ${ }^{1}$ BMW Group, Munich, Germany \\ ${ }^{2}$ Technical University of Munich, Munich, Germany \\ Email:kamil.omozik@bmw.de
}

\begin{abstract}
Summary: Conditionally Automated Driving (CAD) may reduce drivers' mental load and provide the driver opportunities to engage in non-driving related tasks (NDRTs). Such systems can be expected to enter the market within the next few years and effects of automated driving need to be better understood first to maximize their potential benefit. A road-traffic study with $\mathrm{N}=41$ subjects was conducted using a Wizard-of-Oz vehicle to simulate CAD. We observed driver behavior during the initial use of CAD and set out to answer the question: How long does it take to relax? Gaze behavior, seating position, NDRT and self-reported feedback helped in identifying the phases of initial contact and familiarization. The results showed that loose seating position, glance off the road, NDRT engagement and self-reports indicate a familiarization after $10 \mathrm{~min}$ of total CAD and correlated with gender and previous experience with advanced driver assistance systems (ADAS). No significant connection was found between subjective and objective data.
\end{abstract}

\section{INTRODUCTION}

Since CAD systems (Level 3 SAE, 2016) take over the control of both longitudinal and lateral vehicle control temporarily, CAD may increase driver comfort (Payre, Cestac \& Delhomme, 2014) as well as safety (Nunes, Reimer \& Coughlin, 2018; Trimble, Bishop, Morgan \& Blanco, 2014) due to better and quicker decisions made by automation. However, people meet selfdriving cars with both fascination and reservation. The acceptance and openness towards selfdriving cars correlates with both demographics and car use (König \& Neumayr, 2017).

Furthermore, trust in automated vehicles plays an important role for the safety perception and acceptance as well as the decision when to engage the automated system (Forster, Kraus, Feinauer \& Baumann, 2018). For the development of CAD, the driver-system interaction, behavioral changes over time and engagement have to be well understood to create insight into the driver's trust and acceptance.

Currently, driving simulation is used for the majority of CAD studies, as prototypes for on-road testing of subjects in real traffic situations are rarely available. Besides various advantages of driving simulation like objectivity and reliability, there may be constraints of the results' validity (Fisher, Rizzo, Caird \& Lee, 2011). Thus, the current study employed a prototypical vehicle to observe the behavior of participants during CAD. The focus of this paper is the shift from novice (tensed) to experienced (relaxed) users, the point of time when it happens and the factors which might indicate it. .

First CAD road traffic studies postulate that the overall excitement about CAD as well as the glance time on the road decreases over time, drivers engage more in NDRTs, they relax more, and their perceived safety increases (Andersson et al., 2018). However, these studies relied on relatively small-scale data samples, used low speed settings, and had varying weather conditions. 
The goal of this study was to investigate the user adoption effects of CAD and the time it takes for CAD users to show first behavioral changes, as well as the effect of gender, age and previous ADAS experience on this period. Both qualitative and quantitative data were analyzed to identify behavioral changes. .

We hypothesized that the more often participants activate $\mathrm{CAD}$, the sooner they show characteristics of relaxation regarding gaze behavior, seating position and NDRT engagement. Additionally, the longer the duration of CAD, the higher is the percentage of participants who show these characteristics of relaxation.

\section{THEORY}

Behavioral measures are not intrusive and can be collected continually (Hergeth, Lorenz, Krems $\&$ Toenert, 2015). Furthermore, driver's acceptance and interaction with the system are relevant to research relaxation during CAD. Therefore, we chose a multimethod approach to measure self-report and behavioral observation (Forster, Hergeth, Naujoks \& Krems, 2018; Hoff \& Bashir, 2015) that might be used to capture momentary changes in users' behavior and indicate the point of relaxation and familiarization.

Gaze behavior. Gaze behavior provides a direct measure of automation trust during CAD. Hergeth, Lorenz, Vilimek \& Krems (2016) indicate a negative correlation between drivers' selfreported automation trust and the monitoring frequency, which they define as how often the driver cross-checked the CAD system. To quantify the monitoring frequency we measured the driver's glance off the road (any glance off the driving scene) in real time and calculated the longest glance off the road (LGOR) every minute. Additionally, we recorded the time of the maximum LGOR over the whole experiment time. This helped us to identify at what point the driver does not feel a need to monitor the CAD system anymore.

Seating position. A higher shoulder level and head position (z-axis) is considered to indicate stress and discomfort (Beggiato, Hartwich \& Krems, 2018; Tran \& Trivedi, 2010). The driver's foot on or hovering over the brake pedal indicates that the driver feels a need to be ready to take over the maneuvering control (Andersson et al., 2018). Based on these body metrics we classified the driver's seating position by the following categories (Table 1) and identified the point of relaxation. Figure 1 shows examples of the defined seating position categories.
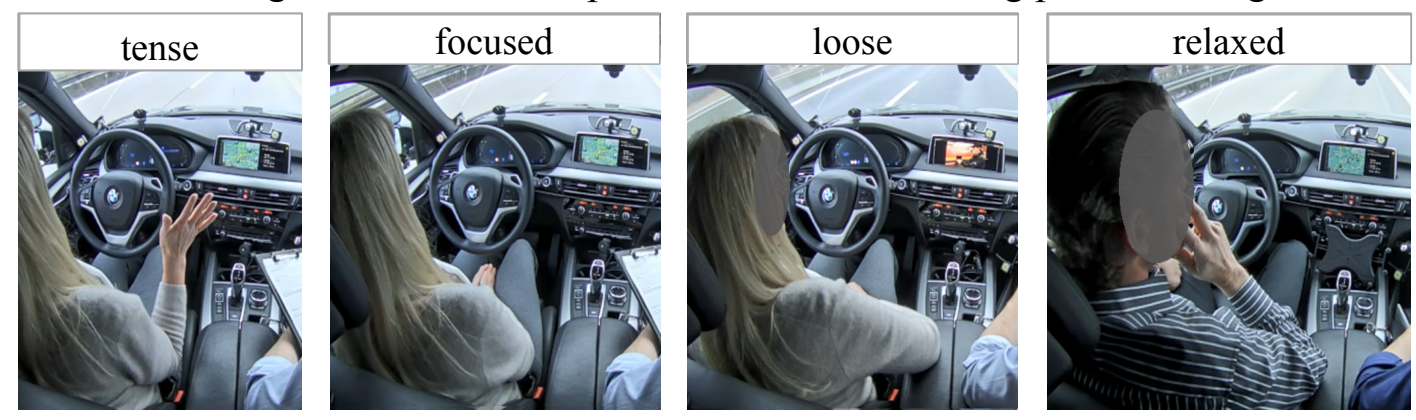

Figure 1. Examples of categories for seating position

Non-Driving Related Tasks. The participants could engage in NDRTs at any time during automated driving. We used the time span from CAD activation until the decision to attend a NDRT to identify the time of familiarization. Andersson et al. (2018) indicate a reduced time to engage in a NDRT at the second encounter with $\mathrm{CAD}\left(\mathrm{M}_{\mathrm{D} 1}=8.2 \mathrm{~min}, \mathrm{M}_{\mathrm{D} 2}=2.0 \mathrm{~min}, \mathrm{~N}=9\right)$. 
This is relevant since subjects experienced during this study a planned interruption of the CAD section and activated afterwards the $\mathrm{CAD}$ for a second time.

Self-reports. We recorded the point of time when participants gave any self-initiated feedback during CAD regarding their relaxation or familiarization (feedback). Additionally, the participants were asked in an interview to estimate and quantify the time it took them to get used to the CAD system after the study (interview): "How long was your familiarization phase?"

Table 1. Categories for seating position

\begin{tabular}{lcccc}
\hline Seating category & Hands Position & Feet/Legs & Head & General posture \\
\hline tense & $\begin{array}{c}\text { close to steering } \\
\text { wheel }\end{array}$ & $\begin{array}{c}\text { hovering } \\
\text { brake pedal }\end{array}$ & upright & $\begin{array}{c}\text { symmetric and facing } \\
\text { driving direction, } \\
\text { large spin extension }\end{array}$ \\
\hline loose & in lap & $\begin{array}{c}\text { upright } \\
\text { pointing at pedal }\end{array}$ & upright & $\begin{array}{c}\text { symmetric and facing } \\
\text { driving direction, } \\
\text { median spin extension }\end{array}$ \\
\hline relaxed & $\begin{array}{c}\text { gesticulating / } \\
\text { laid down }\end{array}$ & $\begin{array}{c}\text { wide } \\
\text { pointing elsewhere } \\
\text { toe on the floor }\end{array}$ & loose & $\begin{array}{c}\text { non-symmetric and facing } \\
\text { driving direction and NDRT, } \\
\text { median spin flexion }\end{array}$ \\
\hline & $\begin{array}{c}\text { laid down / passive } \\
\text { gesticulating }\end{array}$ & $\begin{array}{c}\text { widerossed } \\
\text { random placement }\end{array}$ & tilted & $\begin{array}{c}\text { non-symmetric and facing } \\
\text { NDRT, large spin flexion }\end{array}$ \\
\hline
\end{tabular}

\section{METHOD}

\section{Participants}

Forty-one participants were recruited via mail among the general population and voluntarily took part in the study. None of them had previous experience with CAD. 14 participants were excluded due to irregularities in the proposed route such as temporary construction sites and traffic accidents. The resulting sample size for analyzation $(\mathrm{N}=27)$ consisted of 19 male and 8 female participants between 20 and 65 years old $(\mathrm{M}=41.63)$. The participants owned a driver's license for 23.78 years on average $(\mathrm{SD}=10.81)$. Cruise control $(88 \%)$ and active cruise control (49\%) were the most used ADAS. $52 \%$ of male and $12 \%$ of female subjects were ADASexperienced.

\section{Apparatus}

The study was conducted with a real-vehicle prototype evaluated by Gold, Meyer \& Fischer (2017). The Wizard-of-Oz vehicle (Figure 2) is equipped with cameras for driver and road scene

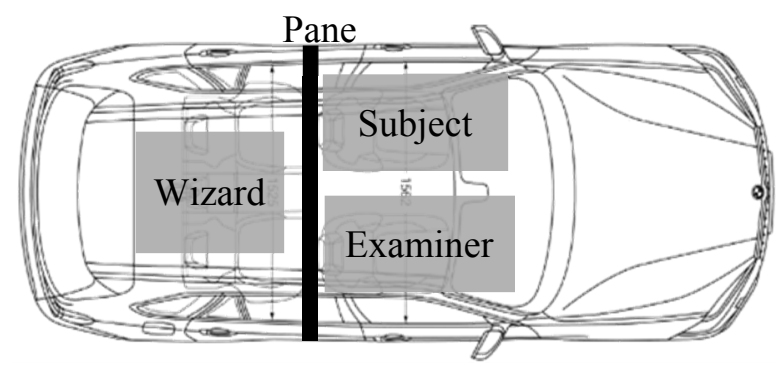

Figure 2. Concept of Wizard-of-Oz vehicle for CAD (Gold, Meyer \& Fischer, 2018) 
observation as well as an acoustic and visual separation pane - a two-way mirror - to hide the driver (wizard) on the backseat simulating the CAD system. The participant on the driver seat is able to steer the vehicle manually as well as activate and deactivate the CAD system (=wizard) on the freeway.

\section{Experimental Design}

The Wizard-of-Oz experiment was conducted on a $60 \mathrm{~km}$ section of a public two-lane freeway outside of Munich between 9 am and $5 \mathrm{pm}$. Each participant was introduced to the test vehicle after the wizard driver was already seated in the backseat. The 90-minute experiments started with low-speed introduction to the CAD. Afterwards the participant drove manually and activated the CAD mode upon entering the freeway. The CAD system was traveling at a maximum velocity of $130 \mathrm{~km} / \mathrm{h}$. During CAD the participants assessed automated maneuvers. The participants were instructed that they would not have to monitor the CAD system while it was active. Furthermore, participants could attend to any NDRT. On the freeway each participant experienced a short CAD introduction (first exposure) of $3 \mathrm{~km}(\mathrm{M}=1: 55 \mathrm{~min}, \mathrm{SD}=0.15 \mathrm{~min}$ ) followed by two CAD sections with a total of $40 \mathrm{~km}$. Section $1(\mathrm{M}=8: 46 \mathrm{~min}, \mathrm{SD}=0.62 \mathrm{~min})$ and Section $2(\mathrm{M}=8: 57 \mathrm{~min}, \mathrm{SD}=1.23 \mathrm{~min})$ were separated by a $9 \mathrm{~km}$ construction site. The participants were asked to take over and drive manually through the construction site $(\mathrm{M}=9: 20$ $\min , \mathrm{SD}=2.46 \mathrm{~min}$ ) and reactivate $\mathrm{CAD}$ after the construction site.

\section{RESULTS}

Driver observation and behavior analysis was video based. A significance level of $\alpha=.05$ was set. All reported correlation analyses were performed using Pearson's r correlation coefficient.

Gaze behavior. The more often CAD was activated, the longer the duration of LGOR was. During first exposure the median was $0 \mathrm{sec}$ (Range $=0-8 \mathrm{sec}$ ). Figure 3 shows the maximum LGOR at 11 mins of CAD ( $\mathrm{Md}=4 \mathrm{sec}$, Range $=1-52 \mathrm{sec})$. The same pattern was found for the starting point of LGOR, in the first section it started on average at 4:58 $\mathrm{min}(\mathrm{SD}=135.3 \mathrm{sec})$ and in the second at 2:29 $\mathrm{min}(\mathrm{SD}=137.6 \mathrm{sec})$ after $\mathrm{CAD}$ activation. Due to the short presentation period, the starting point of LGOR for first exposure was not noted.

Seating position. In the first and second section, participants showed a change in their seating position (Figure 3). The percentage of participants sitting loose increased from $44.4 \%$ to $92.6 \%$. When activating CAD in section 2, most participants stayed in a loose (88.5\%) seating position. After another 4 min drive, every participant showed characteristics of the loose seating positon. The relaxed seating position was only observed by a few participants $(55.6 \%)$ Therefore, the loose posture (3) seems to indicate familiarization. Seating position during first exposure strongly correlated with gender $(\mathrm{r}=-.761, \mathrm{p}<.001)$ and moderately with ADAS experience $(\mathrm{r}=$ $.406, \mathrm{p}=.036)$. The time participants started showing characteristics of the loose $(\mathrm{r}=.725, \mathrm{p}<$ $.001)$ and the relaxed $(\mathrm{r}=.621, \mathrm{p}<.001)$ seating position were strongly correlated with gender.

Non-Driving Related Tasks. During first exposure, $78 \%$ of the participants engaged in NDRT. During the first and second section nearly all participants $(93 \%)$ engaged occasionally in NDRT (see Figure 3). On average participants started engaging 3:59 $\mathrm{min}(\mathrm{SD}=3.93 \mathrm{~min})$ after CAD activation. Engaging in NDRT during first exposure $(\mathrm{r}=-.434, \mathrm{p}=.024)$ and the starting point during section one $(r=.514, \mathrm{p}=.009)$ correlated moderately to strongly with gender. The observed NDRTs in this study were using smartphone or tablet, watching a video on the central vehicle display or reading a newspaper. 
Self-reports. Being asked when they felt familiarized with the CAD system, participants reported on average 6:20 $\mathrm{min}(\mathrm{SD}=4.58 \mathrm{~min})$ of driving. 17 participants gave self-initiated feedback, on average after 11:07 $\mathrm{min}(\mathrm{SD}=6.05 \mathrm{~min})$ of driving with CAD. Previous experience with ADAS correlated moderately with the interview outcome $(\mathrm{r}=-.448, \mathrm{p}=.019)$.

Internal consistence. Behavioral and self-reported data were correlated respectively. The behavioral observations as well as the self-reported data showed moderate to high intercorrelations among themselves (Table1), but not with each other.

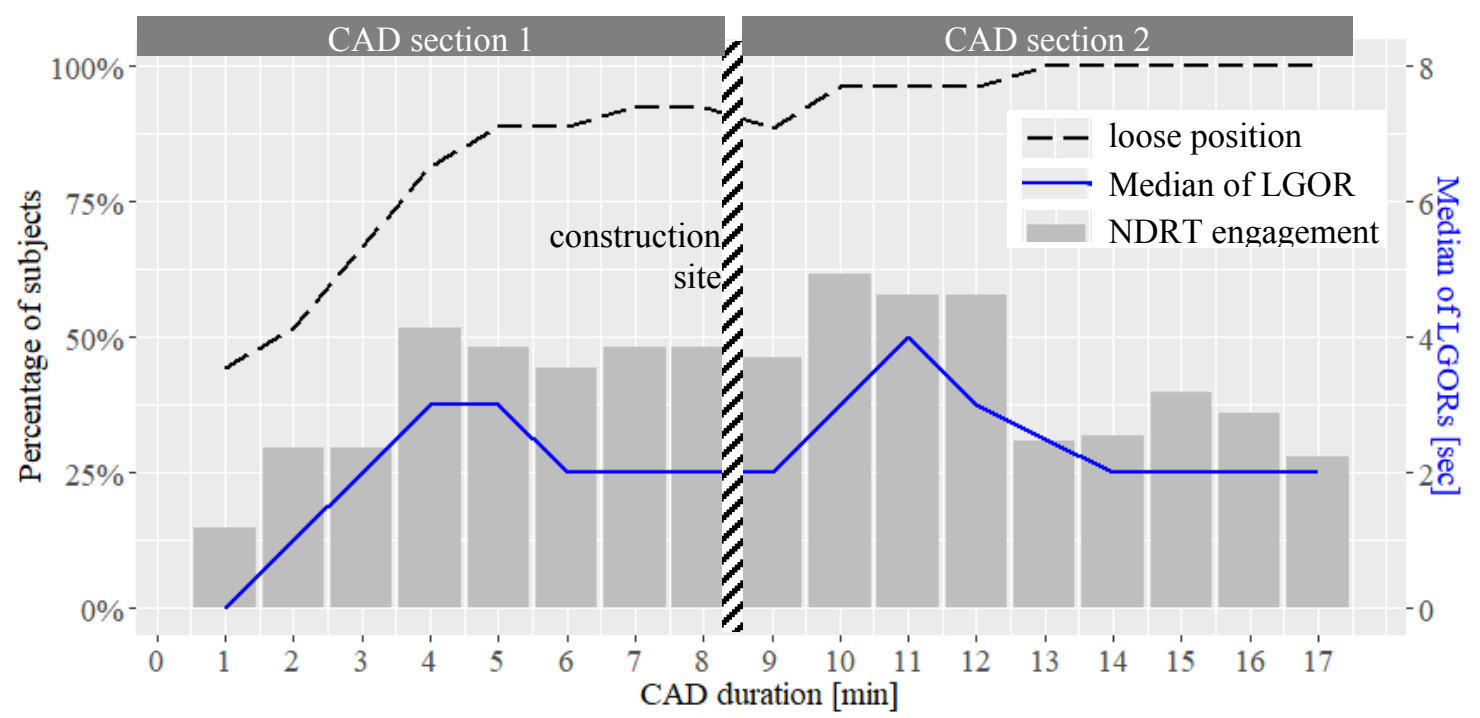

Figure 3. Mean seating, gaze and NDRT behavior over time

Table 2. Correlation matrix of the used behavioral metrics

\begin{tabular}{l|c|c|c|c|c}
\multicolumn{2}{c}{$\begin{array}{c}\text { Point of max. } \\
\text { LGOR }\end{array}$} & $\begin{array}{c}\text { Start of loose } \\
\text { seat. pos. }\end{array}$ & Start of NDRT & \multicolumn{2}{c}{$\begin{array}{l}\text { Self-report } \\
\text { while CAD }\end{array}$} \\
\hline Point of max. LGOR & - & & & & \\
\hline Start of loose seat. pos. & $\mathbf{. 3 8 3 *}$ & - & & & \\
\hline Start of NDRT & $\mathbf{. 3 4 4 ^ { * }}$ & $\mathbf{. 8 0 6 * *}$ & - & & \\
\hline Self-report while CAD & .355 & .244 & .315 & - & \\
\hline Interview & .287 & .002 & .136 & $\mathbf{. 5 3 3 *}$ & - \\
\hline
\end{tabular}

Note. ${ }^{*} p<.05, * * p<.01,{ }^{* * *} p<.001$ (one-tailed)

\section{DISCUSSION}

The familiarization with the CAD system could be observed for observational and self-reported data. On one hand, the longer people drove with $\mathrm{CAD}$, the longer were their observed gazes off the road, the more they sat in a loose seating position and the more they engaged with NDRTs. On the other hand, the more often participants activated the system, the faster they showed signs of relaxed behavior (Andersson, 2018). After around 10 minutes of driving, the relaxation regarding gaze behavior and seating position were highest (Figure 3). However, the defined relaxed seating position seems to be not practical as a measure for familiarization since it could be observed only for some subjects and only temporarily. According to these observational measures, self-initiated feedback was given at roughly the same time. Still, self-initiated feedback did not correlate significantly with behavior measures, which could be due to the wide 
range of feedback timing ( $36 \mathrm{sec}-18 \mathrm{~min}$ ). Interview data did not correspond with the other measurements of relaxation, which could be attributed to effects of social desirability. Interestingly, relaxation characteristics regarding gaze behavior and NDRT engagement flattened out after $13 \mathrm{~min}$. There were no changes in external variables or seating position, possibly because people became bored with their NDRTs. Further studies could investigate more complex NDRTs, since lower LGOR might indicate interest in the system behavior and not a lack of relaxation or trust in CAD. .

Age had no impact on driver behavior. Gender differences were most observable in seating position. During first exposure, women showed more characteristics of the tensed and focused seating characteristics. During the first and second section, men changed earlier to a loose seating position. The same pattern was found for the engagement with NDRT. Women became less engaged during first exposure and started a NDRT later during section one. It seems that previous ADAS experience had an impact on first exposure behavior but not on later use cases. When interpreting the influences of gender and previous experience in ADAS systems on relaxation, it should be noted that the variables confound and male subjects had relatively more prior experience in our sample. .

Overall, response pattern of some subjects indicated that the point of relaxation did not only depend on the total CAD time, but also on the number of experienced CAD maneuvers. This could be further investigated as well.

\section{Limitations}

Since the Wizard-of-Oz vehicle simulated a high performance CAD system and the wizard driver was required to always drive safely and perform a defensive driving style, participants did not experience any malfunctions or potentially dangerous situations. The wizard concept (Gold et al., 2017) allowed a high immersion of participants with high reported levels of trust, comfort and safety. The observations were video based, which include inaccuracy of timing. The threshold to differentiate behaviors are not hard boundaries, which depend highly on the examiner and therefore the result contains certain unavoidable intra- and interindividual differences. We tried to compensate this by training of raters with clearly defined criteria. For further studies, it should be considered that the fourth category of the seating position was not observable for all participants and may need to be redefined to be more sophisticated.

As for all real road studies, a limited generalizability applies regarding road type, traffic density, risk perception and the resulting driving behavior.

\section{CONCLUSION}

The objective of this observation was to investigate the shift from being tense (eyes on the road, tense seating position and without NDRT) to relaxed (eyes off the road, loose seating position and engaging in NDRT) and the time drivers take for such an adaption. The results showed a significant correlation of gender with taking a loose seating position and starting to engage with a NDRT. Male participants tended to be more relaxed during the initial drive, take a loose seating position earlier during CAD, conduct NDRTs earlier and monitor the CAD system less. Taken together, this research provides an insight into driver behavior and its adaption during initial use of a CAD system in real road traffic. 


\section{ACKNOWLEDGMENTS}

We would like to express our very great appreciation to Florian Fischer, Stefan Scheller, Steven Hoffmann, Martin Liebich, Susanna Suchan and Jennifer Schmid for their valuable and constructive work during the planning, conduction and analysis of the mentioned study.

\section{REFERENCES}

Andersson, J., Asker, E., Habibovic, A., Klinegård, M., Lindström, D., Malmsten Lundgren, V. (2018). Method Development for Studies at Test Tracks of Long-Term Behavioral Adaption in Automated Vehicles. SAFER Open Research at AstaZero. Göteborg, Sweden.

Beggiato, M., Hartwich, F., Krems, J. (2018). Using Smartbands, Pupillometry and Body Motion to Detect Discomfort in Automated Driving. Frontiers in Human Neuroscience, 12, 388.

Fisher, D. L., Rizzo, M., Caird, J. K., Lee, J. D. (2011). Handbook of Driving Simulation for Engeneering, Medicine, and Psychology. Boca Raton, FL. CRC Press.

Forster, Y., Hergeth, S., Naujoks, F., Krems, J. F. (2018). How Usability Can Save the Day Methodological Considerations for Making Automated Driving a Success Story. Proceedings of the 10th International Conference on Automotive User Interfaces and Interactive Vehicular Applications - AutomotiveUI '18, Toronto, Canada, 278-290.

Forster, Y., Kraus, J., Feinauer, S., Baumann, M. (2018). Calibration of Trust Expectancies in Conditionally Automated Driving by Brand, Reliability Information and Introduction Videos: An Online Study. Proceedings of the 10th International Conference on Automotive User Interfaces and Interactive Vehicular Applications - AutomotiveUI '18, Toronto, Canada.

Gold, C., Meyer, M.-L., Fischer, F. (2017). Take-Over Performance in a Wizard of Oz Vehicle in Level 3 CAD. Active safety and automated driving, Essen, Germany.

Hergeth, S., Lorenz, L., Krems, J. F., Toenert, L. (2015). Effects of Take-Over Requests and Cultural Background on Automation Trust in Highly Automated Driving. Conference: $8^{\text {th }}$ International Driving Symposium on Human Factors in Driver Assessment, Training and Vehicle Design, Salt Lake City, USA, 331-337.

Hergeth, S., Lorenz, L., Vilimek, R., \& Krems, J. F. (2016). Keep your scanners peeled: Gaze behavior as a measure of automation trust during highly automated driving. Human Factors.

Hoff, K. A., Bashir, M. (2015). Trust in automation. Integrating empirical evidence on factors that influence trust. Human Factors, 57, 407-434.

König, M., Neumayr, L. (2017). Users' resistance towards radical innovations: The case of the self-driving car. Transportation Research Part F: Traffic Psychology and Behaviour, 44.

Nunes, A., Reimer, B., Coughlin, J. F. (2018). People must retain control of autonomous vehicles. Nature 556, 7700, 169-171.

Payre, W., Cestac, J., Delhomme, P. (2014). Intention to use a fully automated car: Attitudes and a priori acceptability. Transportation Research Part F, 27, 252-263.

SAE. (2016). Taxonomy and Definitions for Terms Related to On-Road Motor Vehicle Automated Driving Systems, J3016R.

Tran, C., Trivedi, M. M. (2010). Towards a vision-based system exploring 3D driver posture dynamics for driver assistance: Issues and possibilities. 2010 IEEE Intelligent Vehicles Symposium, San Diego, CA, 179-184.

Trimble, T. E., Bishop, R., Morgan, J. F., Blanco, M. (2014). Human factors evaluation of level 2 and level 3 automated driving concepts (Report No. DOT HS 812 043). Washington, DC: National Highway Trac Safety Administration. 